
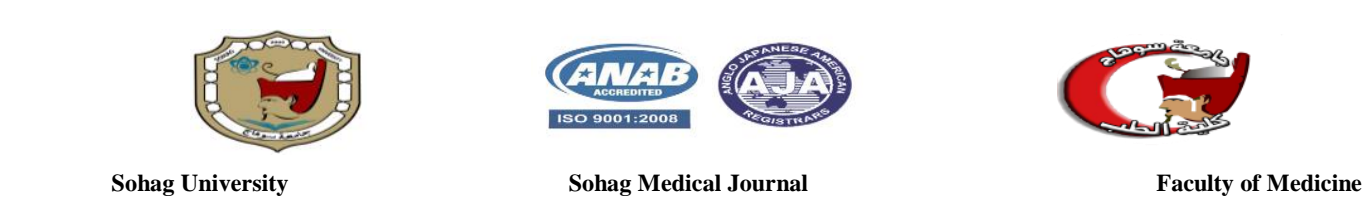

\title{
Role of curcumin in ameliorating hazards caused by prenatal dexamethasone administration on development of the neonates suprarenal glands in albino rats, ( Light microscopic study).
}

\section{Mostafa A.AbdEllah', Salwa M. Ouies², Abeer F.AbdEINnaeem², Walaa N. Elsamman².}

\author{
1. Obstetrics and Gynecology Department, Faculty of Medicine, Sohag \\ University. \\ 2. Human Anatomy \& Embryology Department, Faculty of Medicine, Sohag \\ University
}

\begin{abstract}
:
Introduction: The dexamethasone (Dx) is a synthetic glucocorticoid that passes through the placental barrier especially in late gestation and affects the growth and maturation of most fetal tissues so that The Dx is a commonly used drug in preterm delivery to promote fetal lung growing, lowering the postnatal deaths from respiratory distress diseases.

Aim of work: This study was carried out to study the effects of dexamethasone on the development of neonates' suprarenal glands and the possible protective effect of curcumin against this.

Material \& Methods: In this study, a total of 12 adult pregnant female albino rats were included. The animals were subdivided into 3 groups, five rats for each group. the offspring were sacrificed at birth. The suprarenal glands were processed for light microscope studies.

Results: Variable pathological changes were observed in the adrenal cortex of the dexamethasone-treated group compared to the control one and these changes become little or not present on the curcumin and dexamethasone-treated group.

conclusion: prenatal dexamethasone administration has enormous destructive effects on both the structure and fine structure of the developing adrenal gland. Therefore, it is highly recommended that its use must be restricted to the urgent necessity and in such cases, curcumin as a potent antioxidant can be utilized to ameliorate these adverse effects.
\end{abstract}

Keywords: development, adrenal gland, Dexamethasone, curcumin.

\section{Introduction:}

The suprarenal (adrenal) glands are paired organs that lie immediately above and slightly anterior to the superior pole of each kidney. The glands are surrounded by fat and enclosed within the renal fascia and separated from the kidneys by a small amount of fibrous tissue. ${ }^{(1)}$
Each gland has two parts: cortex and medulla, suprarenal cortex tends to be thicker and has a more yellow appearance due to the high content of fat. the adrenal medulla is reddish-brown in color but there is no sharp anatomical demarcation between the medulla and cortex while these parts have different embryological origins and different fu- 
nctions. ${ }^{(2)}$ The primordium of the cortex is derived from mesodermal tissue, which starts to make steroid hormones during fetal growth, but complete histological and functional differentiation of the three zones of cortex occurs during the neonatal period. ${ }^{(3)}$ The external zona glomerulosa (zg) produces mineralocorticoids hormones as aldosterone; the intermediate zona fasciculata $(\mathrm{ZF})$ produces glucocorticoids hormones like cortisol (corticosterone in mice and rats), and the internal zona reticularis (zr) makes the adrenal androgens in humans and some primates, though not in rats and mice. ${ }^{(4)}$ Chromoblasts originate from the neural crest, then immigrate into the primordium of the suprarenal gland at GD 15 to form the centrally located medulla. ${ }^{(5)}$ Suprarenal Cortical hormones play an important role in multiple physiological processes, as fluid and electrolyte balance, cardiovascular homeostasis, carbohydrates, protein and lipid metabolism; immune and inflammatory responses, sexual maturation, and reproductive function. ${ }^{(6)}$ Due to their potent effect on the maturation of fetal organs, synthetic glucocorticoids are used in human pregnancy who are at risk of pre-term delivery. (7)

Therefore, the Dx is a commonly used drug in preterm labor to promote lung development, lowering the postnatally respiratory distress syndromes and hence decrease postnatal deaths. ${ }^{(8)}$ Despite the multiple favorable effects, exposure to synthetic glucocorticoids during fetal growth may result in intrauterine growth restriction and fetal programming of the hypothalamic-pituitary-adrenal (O$\mathrm{H}-\mathrm{PA}$ ) axis function which is associated with cardiovascular, metabolic, and psychiatric disorders manifested later in life. ${ }^{(9)}$ Curcumin has been used widely to treat injuries and bends, and gastrointestinal, respiratory, and hepatic disorders. (10) Attention to the benefits of curcmin has raised dramatically over the previous decade with numerous cli- nical studies currently researching its efficacy for treating and/or preventing a range of diseases. Many pharmacological studies have been conducted to describe multiple biological actions of curcumin. ${ }^{\text {(11) }}$

\section{Aim of the work:}

This study was carried out to study the effects of dexamethasone on the development of neonate's suprarenal gland and the possible protective effect of curcumin against this.

\section{Materials and methods:}

In this study, a total of 12 adult pregnant female albino rats their weight range from $200-250 \mathrm{~g}$ were used. The animals were brought from the Sohag faculty of science animal house. They were reared under the standard conditions of feeding, light-dark ratio, and temperature, in the Sohag faculty of medicine animal house. Rats were kept in plastic cages in the ratio of 1:4 males and females. Females were examined for vaginal plugs (an indication of the presence of sperm in the vagina) and separated for the experimental protocol and their gestational days were recorded.

\section{The animals were subdivided into three groups with ten animals each: Groups:}

Group I (Control Group): Includes 5 offspring of 4 control mothers injected subcutaneously distilled water.

Group II (Dexamethasone group): Includes 5 offspring of 4 mothers injected subcutaneously with dexamethasone on days 16 and 17 of pregnancy. Dexamethasone ampoules were broug-ht from the Al-America pharmaceu-tical company, the drug preparation was injected subcutaneously in two doses, first on day $16(1.5 \mathrm{mg} / \mathrm{kg}$ body weight $)$ and $(0.5 \mathrm{mg} / \mathrm{kg}$ body weight $)$ on day 17 of pregnancy. ${ }^{(12)}$ 
Group III (Curcumin group): includes 5 offspring of 4 mothers injected first subcutaneously with dexamethasone on days 16 and 17 as mentioned before followed by oral administration of curcumin one hour later at a dose of 15.75 $\mathrm{mg} / \mathrm{kg}$ body weight till delivery of pregnant rat.

Curcumin was purchased from a local market in Sohag and macerated in distilled water, filtered, and orally given daily at a dose of $15.75 \mathrm{mg} / \mathrm{kg}$ body weight. ${ }^{(13)}$ In the three groups, the offspring were sacrificed at birth.

\section{Methods:}

The neonates from each of the groups were randomly selected, sacrificed at birth, The animals were killed by intramuscular IM injection of a mixture of Ketamine ( $90 \mathrm{mg} / \mathrm{kg}$ body weight) and Xylazine $(10 \mathrm{mg} / \mathrm{kg}$ body weight) then their adrenal glands were taken for light microscopic study.

The suprarenal glands were taken at birth, fixed in $10 \%$ formalin for at least $24 \mathrm{hr}$ then processed for light microscopic study, the sections stained with Haematoxylin and eosin (H\&E) for general histological structure. Specimens were examined and photographed in the anatomy department of the fac-ulty of medicine, Sohag University.

\section{Statistical analysis:}

All results were expressed as Mean \pm SD (standard deviation of the mean).

Data were assessed using the Student, s t-test for comparing the means of these variables between different groups.

\section{Results:}

\section{Control group}

Light microscopic examinations of the suprarenal gland reveal the outer conn- ective tissue capsule is formed of many connective cells and fibers. The cortex is thick and composed of the smaller external definitive zone (DZ) and larger internal fetal zone(FZ). The Medulla is apparently demarcated from the surrounding cortex (Fig. 1). The Definitive Zone is formed of small closely packed cells arranged in groups of cells around blood sinusoids. DZ cells have eosinophilic cytoplasm with few tiny vacuoles and large vesicular dark-stained nuclei. The FZ cells are arranged in anastomotic cords (of one to three cell thickness) radially running (towards the medulla). The FZ cells appear with large central nuclei and eosinophilic, vacuolated cytoplasm (Fig.2).

\section{Dexamethasone treated group}

Light microscopic examinations of the suprarenal gland reveal some degenerative changes in the gland in the form of poorly demarcation in between the 2 zones of the cortex, loss of the arrangement of the cortical cells in both the Definitive Zone and the Fetal Zone in comparison with the controls (Fig .3), Some areas of the Definitive Zone shows overcrowding of cells (which extended to the FZ) more than the others and outer relatively thick capsule surrounded the gland. The capsule is composed of cells and fibers with an apparent increase of cellular elements. (Fig.4).

\section{Dexamethasone and curcumin-treat- ed group}

Light microscopic examinations of the suprarenal gland reveal that the gland tissue is nearly or less normal that all degenerative changes that occur due to dexamethasone become less (Fig.5) \&(fig.6). 


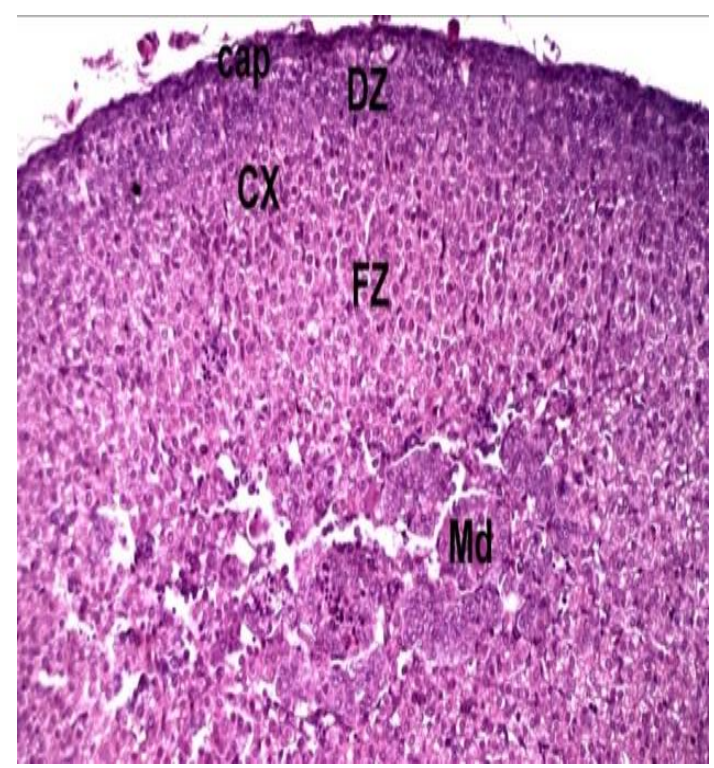

Fig. 1: A photomicrograph of a section of the adrenal gland of the control rat at birth showed: more developed thin capsule (Cap). Cortex (Cx) formed by outer DZ and inner FZ surround a central medulla (Md). $\mathbf{H} \& \mathbf{E}, \mathbf{X 2 0 0}$

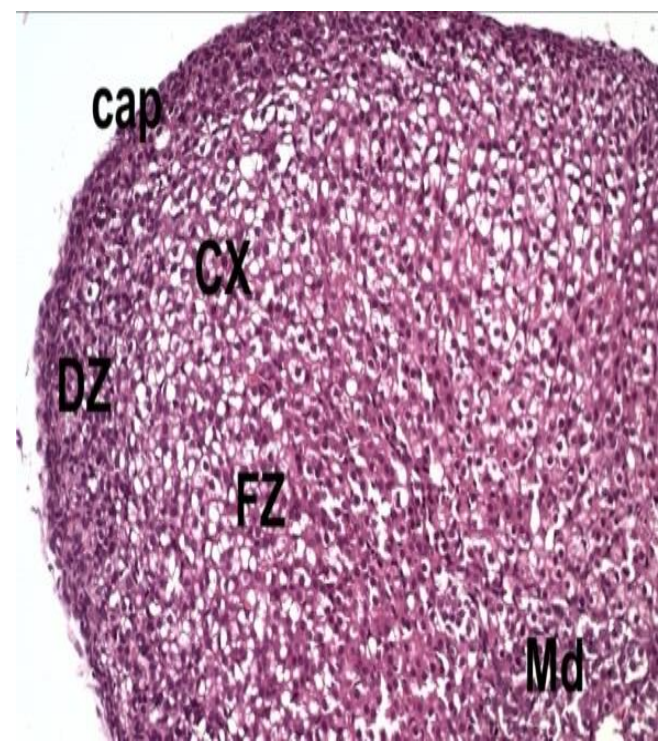

Fig.3: A Photomicrograph of the section of group II (dexamethasone) adrenal gland at birth showing capsule (cap), cortex (CX) formed by DZ and FZ, and medulla (Md), areas of vacations (arrows).H\&E X200.

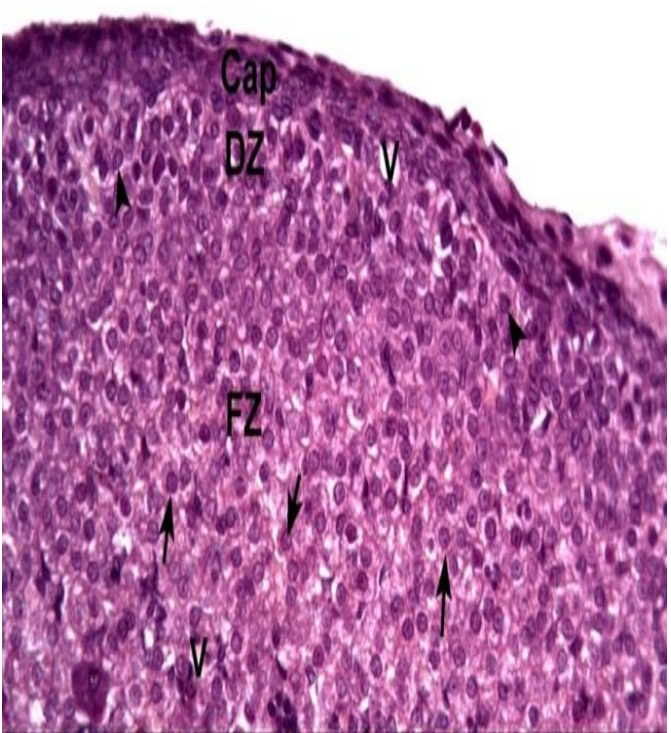

Fig. 2: A magnified part of the previous section showed, outer fibro cellular capsule (cap) outer small dark DZ cells (arrowheads), and large bright FZ cells (arrows) with blood vessels in between (V). H \& E, X400.

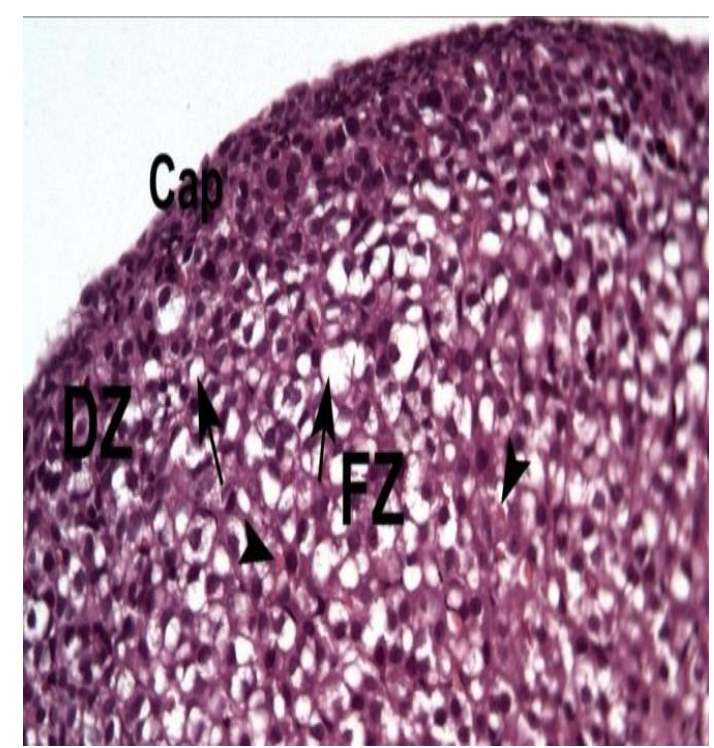

Fig.4: A magnified part of the previous section showing thick capsule (cap), areas of vacuolations in both DZ and FZ (arrows), and areas of hemorrhage in the cortex (arrowhead).H\&E X400. 


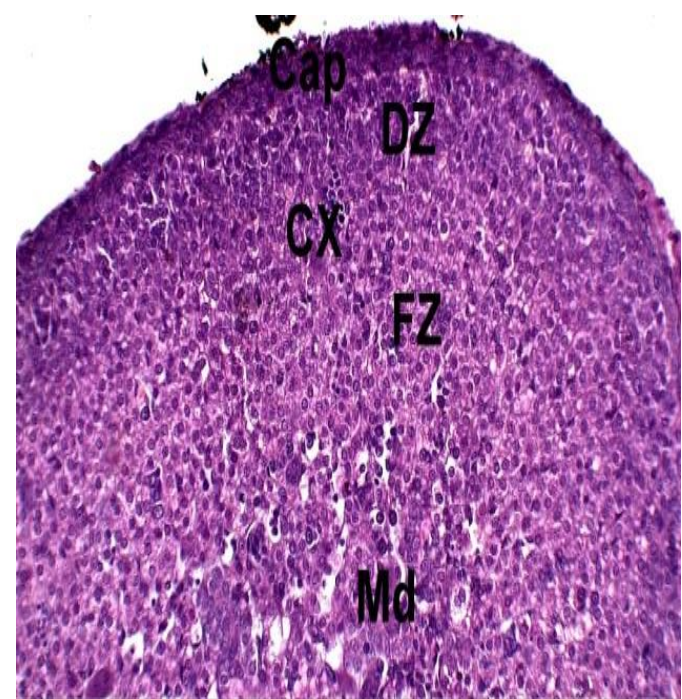

Fig. 5: A photomicrograph of a section of the adrenal gland of group III(curcumin) at birth shows: capsule (Cap). Cortex (Cx) formed by outer definitive zone (DZ) and inner (FZ)surround a central medulla (Md). H \& E, X200.

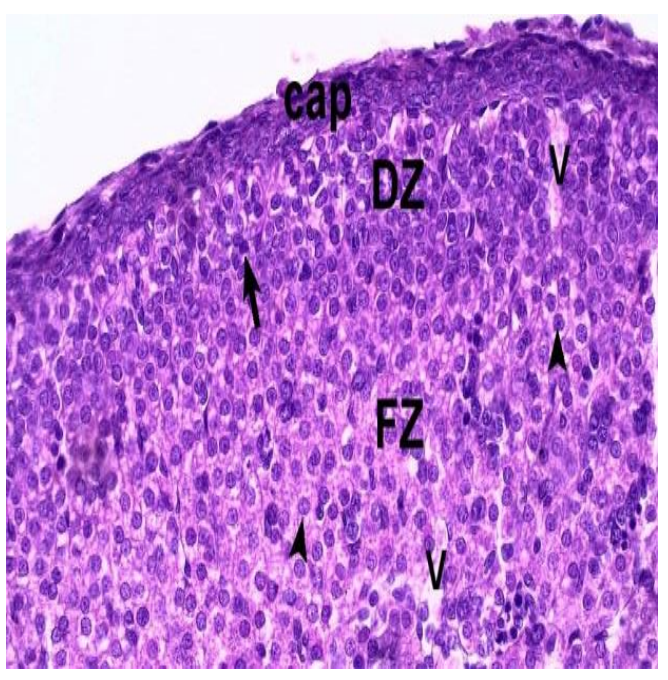

Fig. 6: Amagnified part of previous section showed, near normal fibrocellular capsule (cap), outer DZ cells (arrows)and FZ cells (arrow heads) which appeared normal separated by blood vessels (V) $\mathbf{H}$ $\& \mathrm{E}, \mathrm{X} 400$.

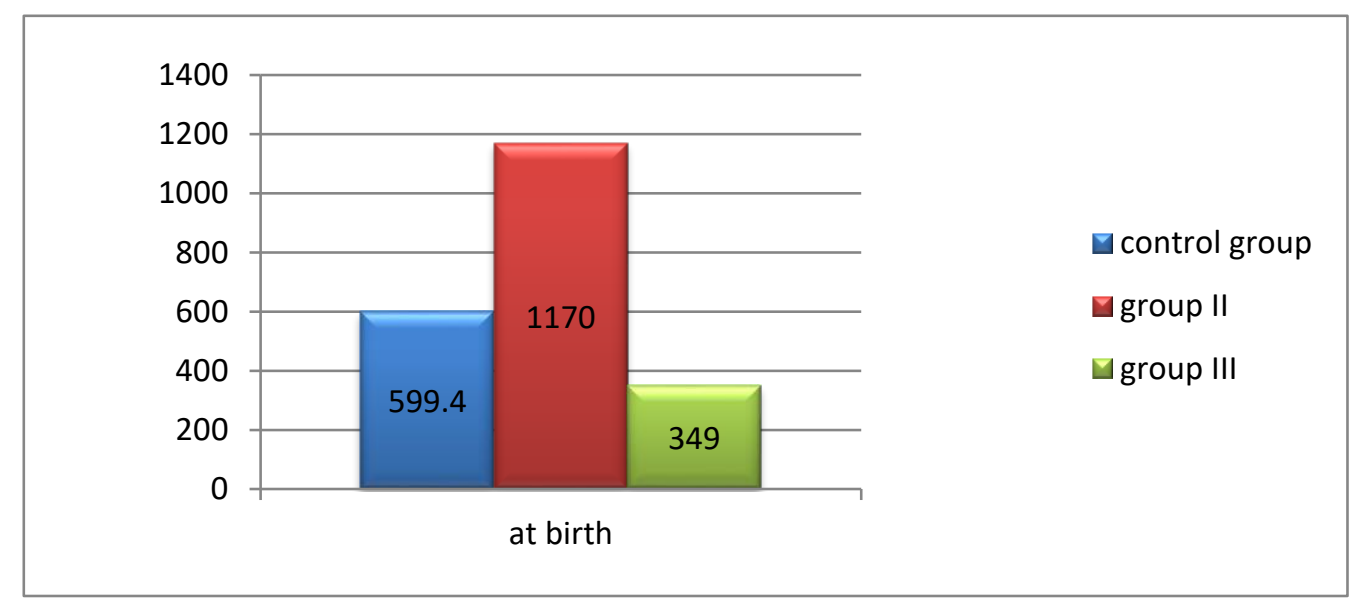

Histogram .1: showing means the thickness of cortex of the three groups at birth.

\section{Morphometric studies:}

The average cortical thickness (arbitrary units, pixels) in the control group was (599.4 \pm 51.6$)$, while in group II it was $(1170 \pm 122.7)$ with a high significant increase ( $p$-value 0.000 ) from the control group, the $3^{\text {rd }}$ group, the thickness was $(349 \pm 20.7)$ with a highly significant decrease ( $\mathrm{p}$-value 0.000 ) from group II, and a highly significant $\mathrm{d}$ ecrease (p-value 0.000) from the control group (Histogram.1).

\section{Discussion:}

The growth of fetal tissues and organs are dependent on many factors, including the hormonal effect. Endogenous glucocorticoids produced by the fetal adrenal glands have an important role in these processes and exert beneficial effects on the growth and maturation where glucocorticoid receptors have been located. ${ }^{(7)}$ It has been reported that prenatal treatment with synth-etic glucocorticoids may exert inappro-prinnately high levels of corticosteroids in the fetus, which leads to restricting fet- 
al growth and increases the risks of disturbance of the metabolic function and endocrine axes, including stress response, growth, and reproduction. (14)

In the present study at birth, the control groups showed adrenal glands in the form of thin fibro cellular capsule, cortex, and medulla. the capsule is formed by fibers and cells but mostly fibers. Definitive Zone cells arranged arches under the capsule. The Fetal Zone cells are arranged in irregular columns with blood vessels in between. Multiple vacuoles (which indicates increase lipid con-tents) were common within the cells of this zone.

These results were in acceptance with ${ }^{(3)}$ that reported that encapsulation of the adrenal primordium the mesenchymal cells from the FZ and this was followed by the development of the DZ cells under the capsule in the prenatal period, this occurs both in humans and rodents. Also (15) demonstrated that the onset of differentiation of the suprarenal gland into cortex and medulla and the synthesis of the steroidogenic enzymes, responsible for hormonal production, occurs during the fetal life in many Mammals. The excretion of adrenocorticotrophic hormone from the fetal pituitary was vital for functional maturation of the suprarenal gland both in fetal and postnatal life. In the rat, fetal encephalectomy leads to atrophy of the adrenal gland. In the present study, the dexamethasone-treated group showed thickened capsules, poor differentiation between cortex and medulla, and loss of control architecture of the adrenocortical cells. The medullary cells showed groups around blood sinusoids with areas of hemorrhage.

These results were in agreement with ${ }^{(7)}$ and (16) who reported that treatment of pregnant rats with Dexamethasone, on the $16^{\text {th }}$ day of gestation, affects the proliferation of fetal adrenocortical cells as well as the ACTH cell of the pituitary gland resulting in increased glucocorti- coid level and marked atrophic changes and suppression of fetal suprarenal function.

In the present study, the suprarenal gland of group III showed preserved architecture and the cells are more or less normal this is in agreement with ${ }^{\left({ }^{(9}\right)}$ who showed that rats injected with corticosteroids followed by curcumin displayed a nearly normal structure of the adrenal gland in relation to control group.

\section{Conclusion:}

Based on the outcome of the present study, it can be concluded that prenatal dexamethasone administration has enormous destructive effects on both the structure and fine structure of the developing adrenal gland. Therefore, it is highly recommended that its use must be restricted to the urgent necessity and in such case, curcumin as a potent antioxidant can be utilized to ameliorate those adverse effects.

\section{References:}

1- Moore KL and Dalley AF: clinically oriented anatomy, Wolters kluwer india Pvt Ltd (2018),p519-522.

2- Llahana $\mathbf{S}$, Follin $\mathrm{C}$, Yedinak $\mathrm{C}$ and Grossman A: Advanced Practice in Endocrinology Nursing(2019).1st edit,p 419-436

3- Xing Y, Lerario A, Rainey $W$ and Hammer GD: development of adrenal cortex zonation. Endocrinol Metab Clin North Am; (2015) 44 (2) pp: 243-274.

4- Wotus C, Levay-Young BK, Rogers LM, Gomez-Sanchez CE and England WC: Development of adrenal zonation in fetal rats defined by expression of aldosterone synthase and 11B- hydroxylase. J. Endocrinol (1998). 139(10):4397-4403.

5-Yamamoto M, Yanai R and Arishima $\mathbf{K}$ : Study of migration of neural crest cells to adrenal medulla by threedimensional reconstruction. (2004)J Vet Med Sci; 66(6): 635-41. 
6-Hart KA, Barton MH, Ferguson DC, Berghaus R, Slovis NM., et al.: SerumFree Cortisol Fraction in Healthy and Septic Neonatal Foals. Journal of Veterinary Internal Medicine (2011), 25(2), 345-355.

7-Manojlović-Stojanoski M, Ristić N, Singh $S$ and Milošević $V$ : Antenatal Treatment with Glucocorticoids and the Hypothalamic-Pituitary-Adrenal Axis. J Med Biochem (2014); 33: 307-16.

8-Ricci F, Salomone F, Kuypers E, Ophelders D, Nikiforou M, Willems M., et al.: in vivo evaluation of the acute pulmonary response to ppractant alfa and bovactant treatments in lunglavaged adult rabbits and in preterm lambs with respiratory distress syndrome. Front pediatr,(2017) 31 (5), pp:186-196.

9-Badawy G: Curcumin ameliorates the hazard effect of prenatal betamethasone administration on the fetal adrenal gland of albino rats. Ejpmr(2018),5(12), 133148

10-Akram M, Uddin S, Ahmed A, Usmanghan K, Abdul Hannan A, Mohiuddin E. et al.,: Curcuma longa and curcumin: A review article. Rom $\mathbf{J}$ Biol - Plant Biol (2010); 55(2): 65-70.

11-Quiles J, Mesa D, Ramirez-Tortosa C, Aguilera C, Battino M, Gil A. et al.: Curcuma longa extract supplementation reduces oxidative stress and attenuates aortic fatty streak development in rabbits. Arterioscler Thromb Vasc Biol(2002); 22(7): 1225-31.
12-Fatthall R, Ahmad S, Mohammed E, Abdulaziz $O$ and Mohamed A: Prenatal and Postnatal Development of the Adrenal Gland of Albino Rats: Light and Electron Microscopic Study. Nature and Science (2018) ;16(5)

13-Hashem M, Atta A, Arbid M, Nada S, Mounir S and Assad G: Toxicological impact of Amaranth, sunset yellow and curcumin as food coloring agents in albino rats. JPMS (2011); 1(2): 43-51.

14-Kurtoğlu S, Sarıcı D, Akin M, Daar G, Korkmaz L and Memur Ş: Fetal adrenal suppression due to maternal corticosteroid use: case report. J Clin Res Pediatr Endocrinol., 2011; 3(3): 160-2.

15-Walker CD and McCormick CM: development of the stress axis: maternal and environmental influences. In: pffaff DW, Arnold AP, Etgen AM, Fahrbach SE, Rubin RT, editors. Hormones, brain, and behavior, 2nd ed., (2009) San Diego: Academic press; pp: 1931-74.

16-Hristic N, Kalafatic, D. Plecas, B and Manojlovic M: The influence of Prolonged Dexamethasone Treatment of Pregnant Rats on the Perinatal Development of the Adrenal Gland of Their Offspring. The Journal of Experimental Zoology, 1997; 279: 5481. 